\title{
Los quechuismos en el mapuche (mapudungu(n)), antiguo y moderno
}

\author{
Gilberto Sánchez $C^{1}$ \\ Universidad de Chile, Chile
}

\begin{abstract}
Resumen
Ya en la época prehispánica los idiomas mapuche (mapudungu(n)) y quechua estuvieron en contacto. Debido a ello, elementos léxicos fueron adoptados por la lengua general de Chile. Los términos quechuas fueron incluidos en los léxicos que forman parte de las gramáticas coloniales del mapudungu(n) (publicadas en 1606, 1765 y 1777), en el Vocabulario Araucano de $1642-1643^{2}$, y también en diccionarios modernos. Algunos términos han dejado de usarse, pero otros se mantienen todavía vigentes en el idioma hablado en el presente. Al parecer, los hablantes no tienen conciencia de su procedencia última. El léxico pertenece a varios campos semánticos (vestimenta, alimentación, etc.), y ha dado origen a formas derivadas. Algunos términos se han incorporado también al español hablado en Chile.
\end{abstract}

Palabras clave: lenguas en contacto, léxico quechua en el mapuche, cultura indígena.

\footnotetext{
1 Para correspondencia, dirigirse a: Gilberto Sánchez Cabezas (gsanchez@uchile.cl), U. de Chile, Facultad de Filosofía y Humanidades, Departamento de Lingüística, Av. Capitán Ignacio Carrera Pinto 1025, Ñuñoa, Santiago, Chile.

2 Atribuido al general holandés Elías Herckmans y publicado por R. R. Schuller (Véase Bibliografía).
} 


\title{
Quechua Borrowings in Old and Modern Mapuche
}

(Mapudungu(N))

\begin{abstract}
Already in pre-Hispanic times the Mapuche (Mapudungu(n)) and Quechua languages were in contact. Due to this, lexical elements were adopted by the general language of Chile. Quechua terms were included in the lexicons that are part of the colonial mapudungu(n) grammars (published in 1606, 1765 and 1777), in the Vocabulario Araucano of 1642-1643, and also in modern dictionaries. Some terms are no longer used, but others are still in force in the language spoken today. Speakers are apparently unaware of their ultimate provenance. The lexicon belongs to various semantic fields (clothing, food, etc.), and has given rise to derived forms. Some terms have also been incorporated into the Spanish spoken in Chile.
\end{abstract}

Keywords: Languages in contact, Quechua lexicon in Mapuche, indigenous culture.

Recibido: 27/03/19 Aceptado: 16/12/19

\section{INTRODUCCIÓN}

Cuando los españoles llegaron a Chile (Diego de Almagro, en 1536; Pedro de Valdivia, en 1540), parte del territorio tradicional formaba parte del imperio incaico. Según el historiador chileno Francisco A. Encina "[...] la primera invasión incaica tuvo lugar alrededor de 1460 de la era cristiana, esto es unos setenta y tres años antes de la llegada de Almagro, bajo el reinado de Tupac Yupanqui, y que solo alcanzó hasta el valle de Coquimbo [unos 500 $\mathrm{km}$ al norte de Santiago]. Veinticinco años más tarde, Huaina Capac, hijo del anterior, mandó nuevos ejércitos, que atravesaron el Maule [240 km al sur de Santiago]. Sufrieron una gran derrota al sur de este río y tuvieron que replegarse detrás de él, fijando su ribera norte como límite de su dominio" (Encina 57-58). Por su parte, Gerónimo de Vivar-el primer cronista de Chile (1558) - escribió "[...] y pasado çierta cantidad de tiempo y años vinieron los yngas, grandes señores del Pirú, y conquistaron con mucha gente esta tierra. Y que estas jentes les administraron y mandaron syguiesen sus rritios y çerimonias, ydolatrasen como ellos lo tenían de costunbre, adorando al sol 
y las piedras grandes a que llaman "guacas"3 (Vivar 53). Era importante, en ese momento, el cacique Quilicanta, quien "por ser valeroso y ser vno de los yngas del Pirú estava puesto en esta tierra por governador" (Vivar 52). La lengua mediante la cual se entendieron españoles y mapuches fue sin duda el quechua. Al respecto, se lee en Vivar "[1]o qual les dio bien a entender [Valdivia] con vn indio que entendía muy bien la lengua [quechua], y el mismo ynga Quilicanta por ser del Cuzco". (Vivar ibid.)

Las lenguas quechua y mapuche estuvieron, pues, en contacto, y la segunda tomó préstamos léxicos de la primera. Los préstamos quechuas ya aparecen consignados en los léxicos de las gramáticas (Artes) coloniales del mapuche, cuyos autores fueron los misioneros jesuitas Luis de Valdivia (Lima 1606), Andrés Febrés (Lima 1765) y Bernardo Havestadt (Münster, Alemania 1777), y también en el Vocabulario Araucano de Elías Herckmann (1642 -1643). Debido a que no existe documentación, no puede precisarse qué léxico procede de la época incaica y cuál pudo ser proporcionado por los indígenas de servicio (yanaconas) que traían los españoles, los cuales eran hablantes de quechua, o, incluso, por los mismos españoles.

Transcurrido el tiempo, algunos de los préstamos cayeron en desuso; otros se mantienen todavía vigentes y han dado origen a formas derivadas (por ejemplo, verbos), según los patrones gramaticales de la lengua. Es imposible saber con precisión cuántos préstamos -pertenecientes a diferentes campos semánticos- provienen del quechua, pues, por una parte, no hay otros léxicos de la época colonial y, por otra, el mapuche actual no ha sido investigado en todos los lugares donde aún es hablado. Parte del léxico quechua se incorporó al español de Chile.

El léxico quechua fue adaptado a los patrones fonológicos y morfológicos del mapuche. Este no posee, por ejemplo, fonemas oclusivos aspirados y eyectivos áfonos; estos fueron sustituidos por $/ \mathbf{p} /, / \mathbf{t} /, / \mathbf{k} /, / \mathbf{c} /$ carentes de tales rasgos (v. gr.: hamk'a $>$ amka). Tampoco posee el fonema fricativo glotal áfono/h/; por consiguiente, fue eliminado (v. gr.: hampi $>$ ampi(n). Respecto de la morfología, en mapuche no ocurren sílabas ni palabras terminadas en /k/, por lo cual debió agregarse una vocal o eliminar una sílaba (v. gr.: kamayok > kamañ). Las formas derivadas se rigen por la morfología del mapuche, v. gr.: challwa-tu-n $(\{-\mathbf{t u}-\}$ morfema verbalizador y $\{-\mathbf{n}\}$ morfema de primera persona de tiempo pasado/presente de modo indicativo) 'yo como/

Otras palabras quechuas contenidas en la obra de Vivar: achupallas, anacona, anchallulla, apo, chacara, çapallo, guanaco, macana, mita, molle, papa, pique-pique, pormocaes, pucaran, quinoa, quipo, quisca, tata, yanacona, ynga, viracocha. 
comí pescado', challwa-tu-pa-n ( $\{-p a-\}$ morfema direccional 'hacia aquí', 'aquí') 'yo vine/vengo a comer pescado'4.

\section{QUECHUISMOS EN EL MAPUCHE (MAPUDUNGU(N) ${ }^{5}$}

Achaw /ačaun/, achawall /ačawaর/. (De atawallpa) s. Gallina, gallo ${ }^{6}$. A) "Atahuallpa. Gallina" (González Holguín I: 36); "atawallpa, la gallina" (Middendorf: 77). B) "Achawal gallina "(Schuller: 12, 22, 39, 56); "Achau, ò achahuall - gallina" Febrés, Calepino: 423); "Achahuall, achaval, gallina" (Havestadt II: 602); "achawall, s., la gallina, el gallo" (Augusta I: 1); "achawall, la gallina" (Alonqueo 1989: 78, 91).

ambi /ambi/. (De hampi) s. Veneno. A) "Hambi - ponçoña" (Santo Tomás II: 290); "Bocado con ponçoña. Hampi" (González Holguín II: 433); "hampi, s. medicina, veneno, filtro" (Middendorf: 480). B) "Ambi, bocado que dan malo" (Valdivia, Vocabulario). La palabra dejó de usarse.

amchi /amči/. (De hamchi) s. Afrecho, salvado. A) "Salvados - hamchi" (Santo Tomás I: 206); "Hamchhi. Afrecho, o buruxo, o el concho assiento de la chicha" (González Holguín I: 145); "Saluados. Hamchi” (González Holguín II: 665); "“hamchi ('hanchi), el afrecho, lo que queda después de estrujada la chicha" (Middendorf: 480); "JAMCHI. 1. Afrecho, residuos del wiñapu o granos empleados para la chicha blanca [...]" (Perroud \& Chouvenc II: 59). B) "Amchi, afrecho" (Valdivia, Vocabulario); "Amchiel afrecho" (Febrés, Calepino: 428); "Amchi, furfur" (Havestadt II: 605); "AMCHI. Afrecho de cebada" (Febrés \& Hernández I: 3). La palabra dejó de usarse.

\footnotetext{
4 También puede significar 'yo vine / vengo a pescar'.

5 Consignamos, en primer lugar, el léxico mapuche con el Alfabeto Mapuche Unificado, uno de los grafemarios empleados para escribir el idioma; luego, entre barras oblicuas, consignamos la transcripción fonológica. No marcamos en las palabras el acento prosódico por no ser distintivo en la lengua. Respecto del quechua, consignamos la forma actual del léxico, según el Diccionario Quechua-Español-Quechua. A continuación de A) incluimos las citas textuales de léxicos del quechua; en B) las citas textuales de léxicos del mapuche. I y II significan la primera y segunda parte, o el tomo o volumen primero y segundo, de una obra, respectivamente. La lista de préstamos del quechua no es exhaustiva.

6 Consignamos los significados que han tenido los préstamos desde la época colonial hasta el presente.
} 
amka /amka/. (De hamk’a) s. Maíz tostado. A) "Hamcca. Mayz tostado" (González Holguín I: 145); “[...] sara 'hank’a, maíz tostado” (Middendorf: 482); "JAMKA. Tostado, sobre todo maíz [...]" (Perroud \& Chouvenc II: 60); "HANK'A. f. Rosetas de granos tostados, grano tostado. Sara hank’a. Tostado de maíz" (Lira: 215). B) "Amca, mayz tostado" (Valdivia, Vocabulario). La palabra dejó de usarse.

ampin /ampin/. (De hampi) s. y v. Medicina, remedio, curar. A) "Hambi emplasto, o medicina generalmente" (Santo Tomás II: 290); "Hambi camayoc - médico, o cirujano generalmente" (Santo Tomás II: ibid.); "Hamppi, o hamppicuna. Qualquiera medicina. Hampi camayoc. El médico o cirujano" (González Holguín I: 145); "'hampi, s. medicina, veneno, filtro. 'hampi camayoj, el droguista, boticario" (Middendorf: 480); "JAMPI. Remedio, medicina" (Perroud \& Chouvenc II: 60); "Hanpi. s. Medicina, remedio, medicamento [...]" (DQEQ $\left.Q^{7}: 135\right)$; "hampikamayoq. s. Médico, persona encargada de los medicamentos [...]" (DQEQ: 136). B) "Ampi, purga o medicina, Ampin, curar, Ampin camañ, médico" (Valdivia, Vocabulario); "Ampin - medicina, ò, remedio, medicinar, curar: ampin lahuen - qualquiera remedio ò hierba medicinal: ampive, ampin camañ, curandero, médico ò machi de ellos" (Febrés, Calepino: 428); "Ampin, curatio, medicina, medela. Item. medeor, medicor, medicinam facere, adhibere, ampin camañ" (Havestadt II: 605-606); "ampin, tr., aplicar remedio de polvo o yerba quemada a la piel, a las llagas" (Augusta I: 7).

apo /apo/. (De apu) s. Gran señor, gobernador, jefe militar. A) "Appo gran señor" (Santo Tomás II: 235); "Apu. Señor grande o juez superior, o Curaca principal, çapay apu, Rey" (González Holguín I: 31); “apu, s. el señor, alta dignidad militar y civil, gobernador, juez; también título de los jefes de familias nobles" (Middendorf: 54); "APU. 1. Gran señor, curaca principal, juez superior: Apu Inka [...]" (Perroud \& Chouvenc II: 11). B) "Apo, gouernador, Apolbin, poner Apoes" (Valdivia, Vocabulario); "Apo Generalissimus, Generalis, A supream [sic] Commander, der allerhöchste Befehlshaber el supremo comandante" (Schuller: 8, 26, 35, 52); "General, ò Governador - Apo, ò thoqui" (Febrés, Vocabulario: 353); “Apo - el Governador, o principal: Apo ghùlmen - el cazique principal de cada Ayllarehue $^{8}[\ldots]$ "... (Febrés, Calepino: 430); “Apo, Superior, Praesul,

Diccionario Quechua Español Quechua.

"Ayllarehue -reducción de nueve parcialidades, con un Apo, y otros ghùlmenes; es como un Ducado, ò Condado" (Febrés, Calepino: 425). "Ghùlmen - Cazique, y hombre rico, y de respeto [...]" (Febrés, Calepino: 490). 
Praepositus, Dux, Praefectus, Tribunus, Centurio, et quicunque alius, qui aliis praeest, imperat praeficitur [...]" (Havestadt II: 607-608)"; "APO. Gobernador, principal" (Febrés \& Hernández I: 3). La palabra dejó de usarse. Se registra en crónicas coloniales y en obras históricas.

awka /aunka/ (De awqa) s. y adj. Enemigo, alzado, rebelde, contrario, montaraz. A) "Aucac - peleador, o enemigo" (Santo Tomás II: 239); "Aucca. Enemigo traydor contrario" (González Holguín I: 38); "auka, s. enemigo, contrario, rebelde, cruel, ingrato, salvaje" (Middendorf: 6); "AUKKA, m. Enemigo, adversario, contrario, rival [...]" (Lira: 71); "AUJA. Adjetivo y sustantivo: enemigo, cruel, guerra, perverso, malo [...]" (Perroud \& Chouvenc II: 14); "awqa. s. Enemigo, contrario, rival, adversario [...]" (DQEQ: 33). B) "aucapu che vfchiduamlay, los aucaes no quieren dar la paz" (Valdivia, Arte: fol. 39); "Auca - alzado, rebelde, ò cimarrón, montaraz: auca chanchu - puerco alzado. Aucan - alzarse, rebelarse" (Febrés, Calepino: 432); "Auca, rebellis [...]" (Havestadt II: 609); "AUCAN. Alzarse, rebelarse” (Febrés \& Hernández I: 4); “auka, adj., alzado, rebelde, muy travieso [...]//-n, n., sublevarse.// s., el alzamiento, la rebelión [...]" (Augusta I: 12); "auka, alzado, no domado, silvestre, no amansado" (Alonqueo 1989: 27); “Awka [aw'ka]: Salvaje; no domado" (Catrileo: 173). Durante la época de la conquista y colonia se denominó aucaes a los llamados 'indios de guerra', es decir, a aquellos que nunca aceptaron la dominación española y se opusieron tenazmente a ella, durante tres siglos. En la actualidad el término se aplica mayormente a los animales montaraces, v. gr., awka kullin /aunka kußinn/, 'animal cerril, cimarrón, no domesticado', pero significa también 'yegua'.

chala /čala/. (De chhalla) s. Paja de maíz. A) "Challa - paja de mayz” (Santo Tomás II: 257); "Chhalla. Caña y hoja de mayz seca" (González Holguín I: 91); "c'halla, s. hojas secas de maíz, paja de maíz" (Middendorf: 370); "CHALA. 1. Maíz empleado como forraje [...]. CHALLA. 1. Chala, maíz empleado como forraje [...]" (Perroud \& Chouvenc II: 27); "chhalla. s. Tallos y hojas del maíz seco que se utilizan como alimento para el ganado [...]" (DQEQ: 83). B) "Chala, paja de mayz seca" (Valdivia, Vocabulario). La palabra dejó de usarse.

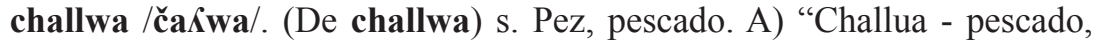
generalmente" (Santo Tomás II: 257); "Pescado. Challhua" (González Holguín II: 629); "challhua, s. el pez" (Middendorf: 341); "CHALLWA. Pez, pescado" (Perroud \& Chouvenc II: 27); "challwa. s. Zool. Pez, pescado" (DQEQ: 46). B) "Challhua, pescado, Challhuantun, pescar, Challhuatuqueúm, pesquería" (Valdivia, Vocabulario); "Challua, Piscis, 
A, Fish, der Fisch, el pez" (Schuller: 12, 26, 40, 57); "Challhua - pescado: challhuan, challhuatun - pescar en general" (Febrés, Calepino: 443); "Challua, piscis challuan, challuatun, piscari, pisces capere, prehendere, trahere" (Havestadt II: 621); "CHALLHUA. Pescado: challhuan pescar: challhuatun comer el pescado" (Febrés \& Hernández I: 8); "challwa, s., el pescado. //-fe, s., el pescador [...] //-n, n., pescar // -tun, n. comer pescado" (Augusta I: 17); "Challwa = el pez" (Alonqueo 1985: 134).

changkan/čạkan/. (De chankay) v. tr. Machucar, despedazar. A) "Machucar cotani.gui. o chancani.gui" (Santo Tomás I: 162); "Chamccani. Frangollar o quebrantar algo moliéndolo" (González Holguín I: 93); "chancay. machacar, moler (papas, chuñu)" (Middendorf: 343); "MACHACAR. Chankay [...]" (Perroud \& Chouvenc I: 92). B) "Chaḡcan, desmembrar" (Valdivia, Vocabulario); "Chagcùn, chagtun - descuartizar, despedazar, trozar" (Febrés, Calepino: 442); “[...] chagcùn, in partes dividere, incidere, in frusta secare, chagtun, chagntun [...]" (Havestadt II: 620). El verbo dejó de usarse y ocurre en español' como chancar 'moler', 'machacar', 'triturar'.

charki /ča.ski/. (De ch'arki) s. Cecina, carne salada en tasajos, secada al sol. A) "Charqui - tassajos" (Santo Tomás II: 259); "Chharqui. Tasajo o cecina o cuerpo seco o el flaquísimo. Chharquini. Hazer tassajo o cecina" (González Holguín I: 98); "ch'arqui, s. carne salada y secada al sol" (Middendorf: 381); "CHARKI. 1. Cecina, carne secada al sol en tajadas menudas [...]" (Perroud \& Chouvenc II: 30); "ch'arki. s. alim. Cecina, chalona, carne salada y seca, a veces congelada [...]" (DQEQ: 98). B) "Charqui, cezina, Charquin, hazer cezina" (Valdivia, Vocabulario); "Charqui - el charqui, ò cezina: charquin - hacerla" (Febrés, Calepino: 444); "Charqui, caro salita, sale condita" (Havestadt II: 622); "CHARQUI. Charqui o cecina [...]" (Febrés \& Hernández I: 9); "charki, s., carne cortada en fajas y secada al sol o ahumada (cecina). //. -n, -tun, tr., charquear (la carne)" (Augusta I: 18). El charqui de carne equina ha sido tradicionalmente muy apreciado por los mapuches. La palabra ha sido adoptada por el español. De ella se ha derivado el verbo charquear, 'hacer charqui', 'herir con arma cortopunzante'.

chawcha/čaunča/. (De chawcha) s. Una variedad de papa. A) "Chhaucha. La papa que madura en breve tiempo" (González Holguín I: 99); "CHÁUCHA, f. Patata aguanosa de crecimiento precoz y de yemas rojizas. Existen también de yemas blancas" (Lira: 106); "CHAUCHA. 1. Papa que madura en poco 
tiempo, papa que se cocina al primer hervor. La chaucha es de calidad inferior [...]" (Perroud \& Chouvenc II: 30); "chaucha. s. Agri. Tubérculo precoz en el crecimiento y producción, como la papa o patata [...]" (DQEQ: 54). B) "Chaucha, papas amarillas (Valdivia, Vocabulario); "Chaucha una laya de papas" (Febrés, Calepino: 444). La palabra dejó de usarse. Se emplea en español y designa una variedad de papa que madura temprano y es pequeña (en Chiloé).

chikta /čikta/. (De ch'eqta) s. y adj. Mitad, medio. A) "[...] Checta. La mitad o vna de dos partes o mas, quimça tahuachecta. Tres o quatro partes" (González Holguín I: 106); "ch'ejta, s., adj. una cosa partida, dividida, pedazo de leña ch'ejtan. La mitad" (Middendorf: 385); "ch'eqta. s. medid. Mitad de la unidad, en medidas de superficie, capacidad y peso [...]// adj. Rajado, partido, dividido" (DQEQ: 102). B) "Chicta, medio peso" (Valdivia, Vocabulario). No aparece documentado en otros léxicos. La palabra dejó de usarse.

chuchoka /čučoka/.(De chuchuqa) s. Maíz tostado o cocido, secado al sol. A) "Chucchucca. Maiz cozido y pasado al sol que es como arroz" (González Holguín I: 118); "chochoka, s. maíz cocido, en seguida helado y al fin secado al sol [...]" (Middendorf: 356); "CHOCHOJA. Comida de maíz molido; hacen secar el maíz al sol, en seguida lo muelen [...]" (Perroud \& Chouvenc II: 36); "chuchuqa. s. Chochoca. Mote reseco. Maíz sancochado, secado al Sol, para posteriormente molerlo y preparar un plato típico de la sierra. SINÓN: Chochoqa" (DQEQ: 71). B) "Chuchoca, ò cunarquen - la chuchoca, esto es, mayz tostado, ò cocido, para secar, y guardar" (Febrés, Calepino: 452); "Maicium coctum, chuchoca" (Havestadt I: 302); "CHUCHOCA. Chochoca, que es el maiz medio asado, o el trigo tostado, para guardarlo" (Febrés \& Hernández I: 11); "chuchoka, s., (=kunárken) maíz tostado o cocido para conservarlo" (Augusta I: 26). La palabra dejó de usarse. Se emplea en español.

chumpi /čumpi/. (De chumpi) s. Faja, ceñidor. A) "Chumbi - ceñidero. Chumbi, o guachuco - faxa para ceñirse" (Santo Tomás II: 273); “Chumpi. Faxa [...]" (González Holguín I: 121); "Faxa angosta de muger. Chumpi" (González Holguín II: 524); "chumpi, s. la faja de los Indios, el ceñidor, hecho de lana de diferentes colores" (Middendorf: 361); "CHÚNPI, f. Faja de primorosos colores para ceñir la cintura con varias vueltas. Franja, cinturón, correa, ceñidor, fajero [...]" (Lira: 143); "CHUMPI. 1. Faja, ceñidor, muchas veces de varios colores y labores, particularmente la faja larga que sirve para las criaturas [...]" (Perroud \& Chouvenc II: 39); "chunpi. s. Cinto o faja, tejida con hilos de color y dibujos primorosos, que sirve 
para fajar a las criaturas y para ceñir la cintura de los adultos [...]" (DQEQ: 75). B) "Chumpi - la faxa, ò ceñidor" (Febrés, Calepino: 454); "Chumpi, fascia, cingulum" (Havestadt II: 628); "chumpi trariwe, s. c., faja de lana con que se ciñen las mujeres y que por su dibujo se llama "chumpi". En las orillas es de color blanco con negro, y en el centro lleva un dibujo de color rojo con amarillo" (Augusta I: 27).

ichu /iču/(De ichhu) s. Paja gruesa. A) "Ychu - yerua, o heno para las bestias" (Santo Tomás II: 299); "Ycchu. Heno, modo de esparto" (González Holguín I: 366); "ic' hu, s. Paja de las punas; pasto más ordinario de las llamas, ovejas y vacas" (Middendorf: 83); "ICHU. 1. Paja de las punas, pasto común de las llamas; sirve para techar [...]" (Perroud \& Chouvenc II: 51); "ichhu. s. Bot. (Stipa ichu). Paja de las punas [...]" (DQEQ: 176). B) "ichu, s., la paja gruesa" (Augusta I: 65).

ichuna /ičuna/. (De ichhuna) s. Hoz. A) "Ychuna - hoz para segar" (Santo Tomás II: 299); "Ychchuna. Hoz" (González Holguín I: 366); "ic huna, s. v. la hoz, la segadera" (Middendorf: 85); "ICHUNA. Hoz [...]" (Perroud \& Chouvenc II: 51); "ichhuna. s. Agri. Hoz, segadera [...]” (DQEQ: 176). B) "Ychuna, hoz" (Valdivia, Vocabulario); "Ichuna - la hoz, o hichona [sic]" (Febrés, Calepino: 519), “ICHUNA. La hoz" (Febrés-Hernández I: 33). La palabra dejó de usarse. Se emplea en español (echona).

kachu /kaču/. (De q'achu) s. Pasto, yerba. A) "Cachhu. La yerua y comida de los animales [...]" (González Holguín: 44); "k'achu, s. yerba del campo" (Middendorf: 288); "PASTO. Jachu [...]" (Perroud \& Chouvenc I: 113); "q'achu. s. Pasto, hierba alimenticia para el ganado y los conejos de indias o qowe [...]" (DQEQ: 492). B) "Cachu, yerua, Cachutun, coger yerua", (Valdivia, Vocabulario); "Cachu herba, das Kraut, la yerba" (Schuller: 13, 22, 58); "Cachu - el pasto del campo" (Febrés, Calepino: 434); "CACHU. El pasto de la pampa" (Febrés \& Hernández I: 5); "Cachu, pastus, pasqua" (Havestadt II: 615); "kachu, s., el pasto, la yerba [...]" (Augusta I: 71); "kachu = los pastos. Hay muchas variedades y son todas medicinales y útiles" (Alonqueo 1985: 137); "kachu [ka'ču]: Pasto; yerba" ( Catrileo: 109).

kamañ /kamap/. (De kamayoq) s. Cualquier oficio. A) "Camayoc -oficial generalmente" (Santo Tomás II: 245); "Camayoc. Oficial o mayordomo, el que tiene a su cargo haziendas, o alguna chacra" (González Holguín I: 48); "Oficial. Camayoc, o el que es diestro abil o sabio en algo" (González Holguín II: 606); "camayoj. el que tiene un empleo, un destino. cama, s. la dignidad, el empleo, la ocupación" (Middendorf: 169); "KAMAYU. Oficial, mayordomo, el que cuida la casa, los intereses del amo [...] Se dice también 
kamayoq: el que está en posesión de algo" (Perroud \& Chouvenc II: 79); "kamay. v. Crear, inventar, modelar, formar, plasmar [...] kamayoq. adj. y s. Especializado, perito, que tiene potestad o dominio sobre algo con total conocimiento de causa [...]" (DQEQ: 197). B) "Camañ, pospuesto significa officio, Cuyulcamañ, carbonero, Yloncamañ, carnicero, Quelletuncamañ, herrador, Quillcacamañ, escriuano, Curacamañ, cantero [...]" (Valdivia, Vocabulario); "Retrave caman Faber ferrarius, A Smith, ein Schmied, un herrero [...]" (Schuller: 8, 26, 35, 56); "Camañ - qualquiera oficio, vg.: chem camañgeymi? qué oficio tienes? ovicha camañ - Ovejero" (Febrés, Calepino: 437); "Camañ, artifex, opifex" (Havestadt II: 616); "CAMAÑ. Oficio, cualquiera que sea: v. gr. Chem camañ nieymi? qué oficio tienes? Ovida camañ. Ovejero: Huaca camañ. Vaquero" (Febrés \& Hernández I: 6); "kamañ, s., guardián, pastor; v. gr.: ofifa kamañ. Ovejero [...]" (Augusta I: 76); "kamañ el pastor, el guardián" (Alonqueo 1989: 130).

kamariku /kamariku/. (De kamarikuy) s. Regalo, obsequio para el español ${ }^{10}$, rogativa. A) "Camaricuk. El que apareja algo, a si mismo se dispone o apareja" (González Holguín I: 48); "camari-ycuy. preparar con cuidado [...]" (Middendorf: 171); "KAMARÍKUY, s. y v. a. Acción de alistar, alistamiento, disposición, preparación. Disponer, alistar, preparar, arreglar, aparejar alguna cosa. v. n. Aparejarse" (Lira: 301); "KAMARIY. 1. Obsequiar, hacer presentes para conseguir protección [...]. 2. [...] kamarikuy: aparejarse, disponerse, prepararse [...]" (Perroud \& Chouvenc II: 79). B) "Camaricu, se llama al tambo donde reciben al español, y lo que le traen de regalo para comer" (Valdivia, Vocabulario); "Camaricu - lo que trahen de regalo al Español: camaricun -hacer este regalo" (Febrés, Calepino: 437); "Camaricu, munuscula, e. g. ova, gallinae, agnus et similia, quae dant Hispanis et Patribus Missionariis, quando eos adeunt praesertim prima vice [...]" (Havestadt II: 616-617); “CAMARICU. Lo que en sus juntas dan de regalo al español, CAMARICUN. Hacer dicho regalo" (Febrés \& Hernández I: 6). En algunas comunidades mapuches se denomina actualmente kamarikun a la 'rogativa' en la cual se pide a la divinidad (ngünechen /yïnečen/ 'el que gobierna a la gente') buen tiempo, cosechas abundantes, etc. La denominación más generalizada es ngillatun /niKatun/ 'pedir', 'rogar'.

kankan /kankan/, kangkan/kaykan/. (De kankay) s. y v. Asado, asar. A) "Cangani, gui. - assar carne u otra cosa" (Santo Tomás II: 247); "Cancani,

10 Implicaba reciprocidad (do ut des). 
cancanahuan. Asar con asador. Canca, o cancasca. Lo asado" (González Holguín I: 49); "canca, s. el asado. cancay, v. tr. asar, tostar" (Middendorf: 176); "KANKAY. Asar; se dice de la carne, mas no del maíz" (Perroud \& Chouvenc II: 80); "kanka. s. alim. Carne asada. Asado [...] kankay. v. alim. Asar la carne u otro alimento [...]" (DQEQ: 198). B) "Cancahue, asador, Cancan, asar en asador" (Valdivia, Vocabulario); "Cancan assa" (Schuller: 27); "Cancan - asado, y asar: cancahue - el asador [...]" (Febrés, Calepino: 438); "Cancan, assum, torridum" (Havestadt II: 617); "CANCAN. Asado y asar. Cancahue. Asador" (Febrés \& Hernández I: 6); "kạkan [kangkan], tr. asar./adj., asado./ s., el asado. // -we, s., el asador" (Augusta I: 73); "kangkan [kay'kan]. Carne asada a las brasas" (Catrileo: 74).

kawitu /kawitu/. (De kawitu) s. Cama de madera, catre. A) "Cama de madera - cullu cabito" (Santo Domingo I: 68); "Cauito. Barbacoa, o cama de madera" (González Holguín I: 52); "KAWITO. Barbacoa, estrado de madera, lecho, catre de palos, cañas, carrizos" (Perroud \& Chouvenc II: 83); "kawitu. s. Catre o lecho rústico y chaclas, hecho para dormir en un lugar apropiado" (DQEQ: 201). B) "Cawyto cubile, A Bedstead, die Bettstette, la cama" (Schuller: 9, 36, 53); "Cahuytu -catre" (Febrés, Calepino: 435); "Cahuitu, lectus" (Havestadt II: 616); "kawitu, s., catre de la cama" (Augusta I: 79); “kawitu [kawi’tu]: Catre” (Catrileo: 68).

killka/kißka/ chillka /čißka/. (De qellqa, qellqay) s. Escrito, carta, papel, libro. A) "Quillca - letra, o carta mensajera. Quillca - libro, o papel generalmente. Quillcani.gui. o quillcacuni.gui. - pintar o escreuir generalmente [...] Quillca camayoc - escriuano o debuxador [...]" (Santo Tomás II: 357); "Quellcca. Papel carta, o scriptura [...] Quellcay camayok. El escriuano de officio, o el gran escribidor [...]" (González Holguín I: 301); "kellka, s. papel para escribir ó dibujar, papel escrito, carta [...] kellkay camayoj, el escribano" (Middendorf: 234); "JELLJA. 3. Escrito, carta, escritura [...]" (Perroud \& Chouvenc II: 67); "qelqay. v. Escribir, graficar, redactar. // Trazar, diseñar [...]" (DQEQ: 456). B) "Quillca, carta, papel, cédula, escriptura, Quillcacamañ, escriuano, Quillcael, escriptura, Quillcahue, pluma para escriuir, Quillcayúm, escritorio, Quillcan, escreuir, Quillcana, escriuanía, Quillcatun, leer, Quillcatuvoe, lector" (Valdivia, Vocabulario); "Chilca epistola, A Letter, ein Brief, una carta" (Schuller: 10, 37, 54), "Quilca, ò chilca - Carta, papel \&c. [...]” (Febrés, Calepino: 615); "Chillca, ò chilca $[. .$.$] carta, papel, escritura [...]. Chillcan, chillcatun -$ escribir" (Febrés, Calepino: 448); "Quillca, chillca, papyrus, charta, epistola, liber" (Havestadt II: 764); "CHILLCA. Carta, papel, escritura [...]” (Febrés \& Hernández I: 10); "chillka, s., libro, carta, noticia [...] //-tun, n. y tr. escribir, leer" (Augusta I: 23). 
kuchi /kuči/. (De khuchi) s. Puerco, cerdo. A) "Puerco, Cuchi” (González Holguín II: 645); "kuchi, s. el puerco, cerdo" (Middendorf: 284); "KHÚCHI, m. Cerdo, chancho, puerco, cochino [...]" (Lira: 498); "KUCHI. 1. Chancho: Kuchiwasi: chiquero [...]" (Perroud \& Chouvenc II: 86); "khuchi. s. Zool. (Sus scrofa domesticus, Gray). Cerdo, chancho, puerco [...]" (DQEQ: 225). B) "Cuchi, el puerco" (Valdivia, Vocabulario); "Cuchy, cuchi Sus A Swine, das Schwein, el puerco cerdo chancho" (Schuller: 11, 25, 39, 56); "Cuchi - dicen en Chiloé al chancho" (Febrés, Calepino: 462); "CERDO. Chancho, sancho, cuchi” (Febrés \& Hernández II: 19). La palabra ocurre en el español de Chiloé, y se emplea para llamar o espantar a los cerdos.

kuraka /kuıaka/. (De kuraka). Señor principal, cacique principal. A) "Curaca - señor principal de vasallos" (Santo Tomás II: 277); "Curaca. El señor del pueblo. Curaca. El señor mayor, o superior [...] El que tiene la voz por todos" (González Holguín I: 55); "curaca, s. (curaj-ka) el jefe de una tribu" (Middendorf: 191); "KURAKA. Señor del pueblo, jefe de pueblos o tribus en tiempos del imperio [...]" (Perroud \& Chouvenc II: 88); "kuraka. s. Hist. Curaca. Autoridad comunal en la época del Imperio del Tawantinsuyu (DQEQ: 212). B) “Curaca, Dominus, Dynasta, A Lord” (Schuller: 8, 26, 35), "Apo curaca A Governor, das Oberhaupt, oder der Heerführer, el cabeza, jefe, cabecilla" (Schuller: 35, 52); "Curaca- usan para decir gran Cacique, y eran criados entre Españoles, y les daban las encomiendas" (Febrés, Calepino: 466); "Curaca, ita olim appellabantur Caciquiorum filii, qui honeste ab Hispanis educati, bonisque moribus instructi, suis praeficerentur" (Havestadt II: 638). La palabra dejó de usarse. Se registra en crónicas coloniales y en obras históricas.

kutama /kutama/. (De kutama) s. Saco, costal, alforja. A) "Cotama arguenas. Cotama, longo - costal, o talega" (Santo Tomás II: 271); "Cutama. Costal" (González Holguín I: 57); "cutama, s. Saco para conservar granos" (Middendorf: 196); "KUTAMA. Costal [...]" (Perroud \& Chouvenc II: 90); "kutama. s. Costal, saco, gangoche" (DQEQ: 215). B) "Cutama - un como saco, ò alforjas, que hacen del poncho" (Febrés, Calepino: 467); "SACO. Sacu, custal, cutama" (Febrés \& Hernández II: 90); "kutama/ s., saco arreglado como alforja. // - kənun, tr., dejar puesto o metido en una cutama" (Augusta I: 101). La palabra se emplea en español rural.

lilpu /lilpu/. (De rirpu, lilpu) s. Espejo, vidrio. A) "Rirpo - espejo" (Santo Tomás II: 348); "Rirpu. El espejo" (González Holguín I: 318); "Espejo. Rirpu, o lirpu" (González Holguín II: 516); "rirpu (lirpu), s. el espejo" (Middendorf: 431); "RIRPU. Espejo [...]" (Perroud \& Chouvenc II: 148); 
"rirpu. s. Espejo. SINON: lirpu [...]" (DQEQ: 525). B) "Lilpu, espejo, Lilputuun, mirarse en el espejo" (Valdivia, Vocabulario); "Lilpu - qualquiera vidrio, ò talco, y lo toman por el Espejo: lilputun - mirarse en él" (Febrés, Calepino: 533); "Lilpu, vitrium, speculum, schistus in finissimas bracteas fissilis" (Havestadt II: 696); "ESPEJO. Lilpu" (Febrés \& Hernández II: 38). La palabra dejó de usarse y, en su lugar, se emplea el español espejo.

michi /miči/. (De michi) s. Gato. A) "Mici, o micito" (González Holguín I: 238); "misi, s. el gato china misi la gata" (Middendorf: 591); "MICHI, m. Gato, género de mamíferos felinos digitigrados [...]" (Lira: 648); "MICHI. 1. Gato [...]" (Perroud \& Chouvenc II: 108); "michi. Zool (Felis catus L.) Gato doméstico [...]" (DQEQ:318). B) "Michi - el gato" (Febrés, Calepino: 553); "michi, felis, catus, aelurus" (Havestadt II: 716). La palabra dejó de usarse. Ocasionalmente se emplea en español.

minkan /minkan/. (De mink'ay) v. tr. Alquilar, contratar para un trabajo con pago en comida y bebida. A) "Minccani. Alquilar persona" (González Holguín I: 240); “mink'ay, v. tr. solicitar en el trabajo la ayuda de otros, prometiéndoles de retornarles el servicio recibido de la misma manera; alquilar" (Middendorf: 588); "MINKAY. 1. Reunir, invitar, convidar para la minka o Minkakuy" (Perroud \& Chouvenc II: 109); "mink’ay. v. Comprometer, contratar bajo palabra amical a una persona para un trabajo [...]" (DQEQ: 324). B) "Mincan - Alquilar gente" (Febrés, Calepino: 554); "minkan*, n., buscar gente para el trabajo y pagar con comida: v. gr.: -ián haré un mingaco, una minga" (Augusta I: 140). En español se emplea minga (del quechua mink'a) que es un trabajo voluntario llevado a cabo por amigos y vecinos de un campesino, con motivo, por ejemplo, de una trilla. Se retribuye con comida y bebida.

miski /miski/, mishki /miški/. (De misk’i) s. y adj. Dulce, miel. A) "Mizque - miel obra de las auejas" (Santo Tomás II: 322); "Mizqui. Miel, açucar, o cosa dulce sabrosa, conseruas, o miel de auejas" (González Holguín I: 244); "misk'i, adj., s. dulce, sabroso, agradable, condimentado, el azúcar" (Middendorf: 591); "MISKI. 1. Suave, dulce, sabroso, bien preparado [...] 3. Azúcar, miel” (Perroud \& Chouvenc II: 110); “misk'i.s. Golosina. Manjar agradable al paladar [...] // adj. Dulce" (DQEQ: 326. B) "Mizque dullin, miel de auejas, mizquilcan, endulcecer" (Valdivia, Vocabulario); "Mifqui, mijqui, ò mizqui - la miel, ò cosa dulce: mizqui dullin - miel de abejas [...] Mifquilcan - endulzar" (Febrés, Calepino: 554); "mifqui, miel, dulce [...]" (Havestadt II: 717); "MIEL de abeja. Misqui llullin" (Febrés \& Hernández II: 61); "mijki, adj., dulce. / s., la miel [...]” (Augusta I: 140). 
mita /mita/. (De mit’a) s. Vez. A) "Vez. Mitta, o cutti, o mitta cuti” (González Holguín II: 691); "Mittayoc. El que trabaja por su tanda o vez. Mittayoc maci. El compañero en la tanda" (González Holguín I: 243); "mit’a, s. lo que vuelve periódicamente, la estación, el turno de trabajo, las capas del terreno; vez" (Middendorf: 592); "MITA. 1. Turno periódico, sobre todo lo dicen en rezo [...] 2. Turno en el trabajo, servicio, agua [...] 3. Época, vez, estación [...]" (Perroud \& Chouvenc II: 111); “mit’a. s. Clim. Estación climática, tiempo, época [...]// Vez, turno, tiempo [...]// Sistema de trabajo forzado a que sometieron los españoles a los de sus colonias en América" (DQEQ:328). B) "[...] quiñe mita una vez [...] aldù mita muchas vezes [...]" (Valdivia, Arte, fol. 49); "Mita - vez [...]" (Febrés, Calepino: 554); "quiñe mita, semel, una vice, mari mita, decies, pataca mita, centies, huaranca mita, millies [...]" (Havestadt I: 155); "Mita, vicis" (Havestadt II: 717); "VEZ. [...] mita [...]" (Febrés \& Hernández II: 105); "mita*, (ant.) s. vez; v. g. ká - (=chuməl) la vez pasada" (Augusta I: 166).

muchan /mučan/. (De much'ay) v. tr. y s. Besar, beso. A) "Mochani. gui. - besar generalmente" (Santo Tomás II: 323); "Muchay cuni, o, muchani. Besar a alguno honestamente con reuerencia, o hazer cortesia, o, bessar la mano" (González Holguín I: 246); "much'ay, v. tr. bezar [sic]" (Middendorf: 602); "MUCH'AY, s. y v. a. Acción de besar, beso. Besar, tocar con los labios alguna cosa. Manifestar el afecto besando" (Lira: 668); "MUCHAY. 1. Besar, ordinariamente por amor sensual [...] 3. Adorar, reverenciar, venerar, honrar, saludar [...]" (Perroud \& Chouvenc II: 112); "much'ay.v. Besar. // Reverenciar, alabar y adorar" (DQEQ: 333). B) "Muchan, besar, Muchan, el beso" (Valdivia, Vocabulario); "Muchan, muchatun - besar" (Febrés, Calepino: 557); "Muchan, osculum, suavium, basium. Item. Osculor, deosculor, oscula figere, imprimere: suaviari, basiari" (Havestadt II: 722). El verbo ha dejado de usarse; en su lugar se emplea peshitun (del español besar).

muti /muti/. (De mut'i) s. Maíz o trigo cocido. A) "Mutti. Mayz cocido, o trigo, o y mani" (González Holguín I: 253); "mut'i, s. mot’i, maíz cocido" (Middendorf: 610); "MOTE. Maíz cocido [...]" (Perroud \& Chouvenc II: 112); "MUTI. Mote [...]" (Perroud \& Chouvenc II: 115); "mot'e. s. alim. Mote. Maíz fresco o seco sancochado o hervido, muy utilizado en la alimentación andina. SINÓN: mut'i'" (DQEQ: 332). B) "Muti, mayz cozido, Mutin, cozer mayz" (Valdivia, Vocabulario); "Muthi , ò muti, el mote de maíz, ò trigo cozido" (Febrés, Calepino: 559); "muti, motte, Triticum, maicium coctum" (Havestadt II: 723); "MUTI, o MUCHI. El mote de trigo cocido" (Febrés \& Hernández I: 46). Al parecer, la palabra ha dejado de usarse; en su lugar se emplea kako. Mote se emplea en español. 
pataka /pataka/. (De pachak) adj. Cien, ciento. A) "Pachac - ciento, número" (Santo Tomás II: 333); "Pachac. Ciento" (González Holguín I: 270); "pachaj. Ciento" (Middendorf: 644); "PACHAQ. Cien" (Perroud \& Chouvenc II: 125); "pachak. adj. num. card. Cien, cien unidades, ciento, diez decenas" (DQEQ: 374). B) "Pataca, ciento" (Valdivia, Vocabulario); "Maripataco centum, A hundred, hundert (cien) ciento" (Schuller: 14, 41, 58); "Pataca - ciento: patacan - haver, o tener ciento" (Febrés, Calepino: 581); "Pataca, centum, centuria. patacan, esse centum" (Havestadt II: 738); "PATACA, o quiñe pataca. Ciento [...]" (Febrés \& Hernández I: 53); "pataka, num., ciento. Epu pataka - doscientos" (Augusta I: 166); "100 C pataka un ciento o cien" (Alonqueo 1989: 51).

pataran /pata.ıan/. (De pataray) v. tr. Doblar, plegar. A) "Patarani. gui. - doblar alguna cosa generalmente" (Santo Tomás II: 337); "Patarani o taparani. Doblar ropas" (González Holguín I: 281); "pataray, v. tr. doblar, plegar" (Middendorf: 655); "PATÁRAY, s. y v. a. Plegadura, dobladura, acto de plegar o de doblar [...]"(Lira: 754); "PATARAY. Doblar la ropa [...]" (Perroud \& Chouvenc II: 132); "pataray. v. Doblar, plegar, doblillar las telas o láminas [...]" (DQEQ: 390). B) "Pataran, doblar" (Valdivia, Vocabulario); "Pataran - plegar, doblar" (Febrés, Calepino: 581); "Pataran, plicare, complicare" (Havestadt II: 738); "pataran, tr., doblar (ropa o cualquier cosa)" (Augusta I: 166).

pinkullu /pinkuKu/. (De pinku(y)llu) s. Flauta. A) "Pingollo o gayta" (Santo Tomás II: 338); "Pincullu. Todo género de flauta" (González Holguín I: 286); "pincullu, s. flauta de los Indios menos gruesa que la quena" (Middendorf: 659); "PINKULLU. Flauta grande de 3 a 5 agujeros; para tocarla la colocan horizontalmente [...]" (Perroud \& Chouvenc II: 136); "pinkuyllu. s. Mús. Instrumento musical aerófono hecho de caña delgada de 50 a 70 cms., con embocadura de bisel (como la flauta), muy usado en la música autóctona, en especial en las fiestas carnavalescas [...]" (DQEQ: 399). B) "Pincullu, flauta, Pincullutun, tañer flauta" (Valdivia, Vocabulario); "Pincùllhue, ò pithucahue - una flauta de ellos: pincùlltun, pithucan tocarla" (Febrés, Calepino: 595); "Tibia [...] pincùllhue [...]" (Havestadt I: 512); "Pinkullwe: "[...] Es el pífano mapuche que tiene 4 orificios en un extremo y en el otro solo tiene uno" (Alonqueo 1985: 121).

pirka /pi.ska/. (De pirka) s. Pared, muro. A) "Pircani.gui. o pircacuni. gui. - edificar" (Santo Tomás II: 339); "Pircca. Pared. Pircani. Hazer pared edificar" (González Holguín I: 287); "pirka, s. rumi pirka, tica pirka, t'uru pirka el muro, la pared, de piedra, de adobes, ó barro" (Middendorf: 660); "PIRJA. Pared [...] Dicen también: Pirka" (Perroud \& Chouvenc 
II: 136); "perqa.s. Pared, muro, valla cercada de piedras u otro material de construcción [...]" (DQEQ: 394). B) "Pirca, pared, Pircan . 1. pircatun, hazer pared" (Valdivia, Vocabulario); "Pylca Paries murus A wall eine Mauer una pared" (Schuller: 8, 27, 36, 53); "Pirca - pared; pircan, pircatun - hazerla" (Febrés, Calepino: 596); "Pirca, paries" (Havestadt II: 748); "PARED. Pirca: hacerla, pircan, pircatun" (Febrés \& Hernández I: 71). La palabra dejó de usarse. Se emplea en español.

piskoytu /piskoitu/. (¿De pisqoynu? $\left.{ }^{11}\right)$ s. Trompo, peonza. A) "Trompo o peonça -piscoyño" (Santo Tomás I: 221); "Peonça, juego de niños que la açotan. piscoyñu" (González Holguín II: 623); "piskoinu, s. el trompo de madera" (Middendorf: 701). B) "Pizcoytu, peonça, juego de niños" (Valdivia, Vocabulario); "Pizcoytun, ò piroytun, pijcoytun - jugar al trompo, ò peonza: pizcoytu, pifcoytu, ò piroy - la peonza" (Febrés, Calepino: 598). La palabra dejó de usarse.

porongo /posono/. (De purunku) s. Cántaro (pequeño). A) "Porongo ampolla para bever" (Santo Tomás II: 341); "Purunccu. Vaso de barro cuelli largo" (González Holguín I: 298); "puruncu, s. (poronco) vaso de barro con cuello largo y angosto" (Middendorf: 675); "PURUNKU. Vaso de barro cuellilargo, o Porongo" (Perroud \& Chouvenc II: 142). B) "Purunco - el porongo, cantarito" (Febrés, Calepino: 604); "Cantharus niger, purunco" (Havestadt I: 412). La palabra se emplea en español.

puchun /pučun/. (De puchu) v. y s. Sobrar, sobra(s), resto (de cigarro). A) "Puchu - demasía o sobra" (Santo Tomás II: 342); "Puchucun o puchun. Sobrar alguna cosa" (González Holguín I: 293); "puchu, s. lo que sobra, el residuo, el sedimento, resto, puchuy, v. intr. sobrar" (Middendorf: 667); "PÚCHU, f. Sobra, resto, residuo o exceso que queda del uso de alguna cosa. Lo que queda de la comida" (Lira: 762); "PUCHU. Sobra, resto, cabo: Cera puchu: cabo de vela [...] PUCHUY. Sobrar [...]" (Perroud \& Chouvenc II: 139); "puchu. s. Resto, residuo, margen, sobrante, remate, saldo [...] puchuy. v. Restar, sobrar, exceder, quedar [...]" (DQEQ: 404). B) "Puchun, sobrar" (Valdivia, Vocabulario); "Puchu - las sobras, y la del cigarro. Puchun - sobrar" (Febrés, Calepino: 603); "Puchun, supero, suppeto; superesse, superfluere, satis superque habere, puchu, quod superat, superflua, reliquiae" (Havestadt II: 757); "PUCHU. Sobras de cigarro o de otra cosa. PUCHU Thipa. Sobras de cualquiera cosa. PUCHUN o

11 Quizá provenía de una palabra regional pisqoytu. No la hemos encontrado documentada. 
puchutripan. Sobrar" (Febrés \& Hernández I: 60); "puchu/ln, tr. dejar sobras $[\ldots] / /$-n., n., sobrar $[\ldots]-\mathbf{n}$, n. cigarro la colilla de cigarro $[\ldots]$ " (Augusta I: 188).

pukuchu /pukuču/(De pukuchu) s. Vejiga. A) "Puccuchu, o yspay puru. La bexiga" (González Holguín I: 294); "PUKUCHU. 1. Vejiga de chancho; de cualquier animal, aun del hombre. Algunos hacen secar la de chancho para jugar o guardar su dinero, coca, otras cosas" (Perroud \& Chouvenc II: 140); "pukuchu [...] // Anat. Vejiga" (DQEQ: 407). B) "Pucuchu, vejiga" (Valdivia, Vocabulario); "Pucuchu - la vegiga, con que echan ayuda"(Febrés, Calepino: 603); "Pucuchu, clyster" (Havestadt II: 757); "PUCUCHU. Bejiga con que hechan ayudas" (Febrés \& Hernández I: 60). La palabra ha dejado de usarse; actualmente se dice püfadkon/pïfaøkon/. Por metátesis dio en español copucha, según Lenz: 211. Véase también DUECh: 288.

sasin /sasin/. (De sasiy) v. intr. Ayunar. A) "çacini, gui. - ayunar generalmente, o comer dieta. Çacini, gui. - ayunar por deuocion" (Santo Tomás II. 241); "Çacicuni, o çacini. Abstenerse de algún manjar, obras, vicios, y deleytes" (González Holguín I: 74); "Çaciy. La dieta, o abstinencia" (González Holguín I: 75); "sasiy, v. int. ayunar" (Middendorf: 762); "SÁSSIY, s. y v. a. Acto y efecto de guardar temperancia o abstinencia. Ayunar, guardar temperancia, moderarse en la comida o bebida" (Lira: 884); "SASIY. 1. Ayunar. Sasina punchau: Día de ayuno [...]" (Perroud \& Chouvenc II: 155); "sasiy. v. Ayunar, privarse de los alimentos o bebidas" (DQEQ: 552). B) "Çacin, ayunar" (Valdivia, Vocabulario). El verbo dejó de usarse; en su lugar los misioneros emplearon ayunan (de ayunar), verbo que está vigente actualmente.

suysun /suisun/. (De suysuy) v. tr. Cernir, colar. A) "Cernir - suysuni. gui" (Santo Tomás I: 92); "Suysuni. Colar cosa líquida, o cernir o ahechar con harnero" (González Holguín I: 333); "suisuy, v. tr. cernir, colar. suisuna, s. v. la criba, el cernidor" (Middendorf: 786); "SUYSUY, s. y v. a. Acto y efecto de cernir alguna cosa pulverizada. Cernir, cerner [...]. Colar, pasar un líquido por el colador. Tamizar" (Lira: 948); "SUISUY. Cernir, colar, tamizar, cribar [...]" (Perroud \& Chouvenc II: 162); "suysuy. v. Cernir, colar, tamizar, cribar, zarandear [...] suysuna. s. Cernidor, colador, tamiz" (DQEQ: 594). B) "Çuyçuycun, cerner, Çuyçuyhue, cedaço de españoles" (Valdivia, Vocabulario). La palabra dejó de usarse; actualmente se dice chaytun /čaitun/

tika /tika/. (De tika) s. Adobe, ladrillo. A) "Tica - ladrillo. Ticani.gui. hazer ladrillos" (Santo Tomás II: 363); "Tica. Adobe. Ticani ticacta rurani. 
Hazer adobes" (González Holguín I: 340); “tica, s. el adobe sañu tica el ladrillo" (Middendorf: 817); "TIKA. 3. Adobe, ladrillo, marqueta, molde [...]" (Perroud \& Chouvenc II: 170); "tika. s. [...]// sustancia pastosa moldeada y después endurecida, como el adobe, ladrillo, teja, etc. [...]" (DQEQ: 620). B) "Tica, adobe, Ticahue, adobera, Ticalcan, hazer adobes" (Valdivia, Vocabulario); "Tica, ò thica - adobe: ticahue - adobera. Tican, ticatun - hacer adobes" (Febrés, Calepino: 631); "Later crudus, laterculus ad solem exsiccatus, sed non coctus aut igne tostus, tica" (Havestadt I: 406), “Tica, lateres crudi, sicci” (Havestadt II: 778). La palabra dejó de usarse.

tingiri /tini.xi/. (De tingri, t'inri) s. Enano. A) "Omoto o tingri - hombre enano" (Santo Tomás I: 330); "Ttinri. Enano” (González Holguín I: 343), "t'inri, s. hombre bajo y grueso, enano" (Middendorf: 850); "T'INRI, m. Ser abultado y enano [...]" (Lira: 1009), "TINRI. 1. Enano, ético, gente o animal" (Perroud \& Chouvenc II: 171); "t'inri. adj. Persona o animal de estatura baja y rechoncho [...]" (DQEQ: 668). B) "Tingri, enano" (Valdivia, Vocabulario); "Tingiri - enano" (Febrés, Calepino: 631). La palabra dejó de usarse. Actualmente se dice püchiwitran che /pïči wit' ${ }^{r} a n$ če/ 'persona de baja estatura'.

titi /titi/. (De titi) s. Plomo, estaño, zinc. A) "Estaño, metal - titi" (Santo Tomás I: 133); "Plomo, metal conocido - titi, o tite" (Santo Tomás I: 189); "Titi. Plomo. Yurak titi. Estaño" (González Holguín I: 344); "titi, s. el plomo. Yuraj titi el estaño" (Middendorf: 822); "TÍTI, m. Plomo, metal muy pesado de color azul gris [...]" (Lira: 976); "TITI. 1. Plomo: yuraq titi: estaño [...]" (Perroud \& Chouvenc II: 172); "titi. s. Miner. Plomo, metal dúctil y maleable de color azulino [...]" (DQEQ: 624). B) "Titi, estaño, Tititun, estañar" (Valdivia, Vocabulario); "titi stannum, plumbum, Tin, das Zinn, el estaño" (Schuller: 7, 22, 34, 50); “Titi, ò thithi - el plomo y estaño. Titiln, tititun - estañar" (Febrés, Calepino: 631); "stannum. titi" (Havestadt I: 248), "Titi, plumbum [...]" (Havestadt II: 778); " TITI. Plomo, estaño. TITITUN. Estañar" (Febrés \& Hernández I: 69); "plomo. m. titi [...]" (Augusta II: 292); "titi [ti'ti]: Zinc o estaño" (Catrileo: 129).

tupu /tupu/. (De tupu) s. Medida, legua; prendedor (de aguja). A) "Topo medida" (Santo Tomás II: 365); "Tupu. Medida de cualquiera cosa. Tupu. Legua" (González Holguín I: 347); "Tupu. El topo con que prenden las indias la saya. Tupuni. Medir algo con vara, o medida" (González Holguín I: ibid); "tupu, s. la medida en general, respecto a distancia, granos, líquidos y géneros [...] tupu, s. una legua" (Middendorf: 828); "tupu, s. un gran alfiler de pelo: cuchara con manga en punta, que sirve a las Indias para prender su rebozo (llijlla)" (Middendorf: 828-829); “TÚPU, Medida agraria consistente 
en 44 varas de ancho, por 88 de longitud [...]". TÚPU, m. Prendedor de plata, oro, cobre, etc. que remata en una cuchara, en motivos domésticos, o de la flora, fauna, agrícola, etc., según cada región" (Lira: 985); “TUPU. Prendedor de plata, cobre, oro para sujetar la manta en el pecho [...] 2. Medida, unidad de medida: un tupu de tierra, una vara [...] 3. Legua" (Perroud \& Chouvenc II: 176); "tupu. s. Prendedor fabricado de diferentes metales, principalmente de plata, oro con engastes de piedras preciosas $[\ldots] / /$ Medida, en general// Unidad de medida, de área o de longitud" (DQEQ: 638). B) "Tupu - las leguas de ellos sin medir, y a vezes una equivale a 3 ò 5; quiñe tupuy - una legua hay: epu tupuy - dos hay" (Febrés, Calepino: 632); "Tupu - it: llaman unas ahajas [alhajas] grandes con una plancha redonda de plata como una ostia, ò mayor, con que prenden las mugeres sus mantas" (Febrés, Calepino: ibid.); "Leuca, tria passuum millia, tria miliaria; tertius lapis, tupu" (Havestadt I: 502); "Laminae rotundae argenteae una cum stylo ferreo, quo vestem ad pectus prehendunt, tupu" (Havestadt I: 298); "təpu, s., (= tupu) pieza de adorno de mujeres que consiste en un disco de plata ahuecado algo en el medio, como plato, y provisto de una aguja para clavarlo como prendedor en el pecho" (Augusta I:215). Tupu dejó de usarse como medida itineraria.

ulku /ulku/. (De unku) s. Camiseta, manta, poncho. A) "Camisa de varón huncu o cusina" (Santo Tomás I: 69); "Vncu, Camiseta de indios" (González Holguín I: 355); "uncu, s. vestido de los indígenas, especie de saco o camisa sin manga ni cuello, de género de lana de color colorado ó amarillo, que se lleva sobre el cutis" (Middendorf: 139); "UNKU.1. Camiseta de los indígenas de doble cuello [...]" (Perroud \& Chouvenc II: 179); "unku.s. Hist. Camiseta utilizada en el inkario, con diferentes variantes, como por ejemplo: larga, corta, cosida íntegramente o tan solo medio cuerpo y suelta en el resto, con pequeña manga o sin ella, con flecos o sin ellos, etc." (DQEQ; 685). B) "Ulcu, axo de india, ulcutun, vestirse el axo que es manta interior" (Valdivia, Vocabulario); "ulcu - camiseta, ò poncho con dos listitas no más: ulcutun - ponérselo" (Febrés, Calepino: 668); “Ulcu, vestis" (Havestadt II: 794). La palabra dejó de usarse.

uminta /uminta/. (De humint'a) s. Pan de maíz. A) "Huminta. Bollicos de mayz como tamales. Humintani. Hazerlos" (González Holguín I: 201); "'humint'a. s. especie de torta hecha de una masa de maíz fresco machacado" (Middendorf: 505); "JUMINTA. Bollico de maíz como tamal, envuelto en hoja de mazorca" (Perroud \& Chouvenc II: 74); "humint'a. s. alim. Tamal de maíz tierno y condimentado con dulce, envuelto en p'anqas (hojas de choclos) y cocinado al vapor [...]" (DQEQ: 169). B) "Uminta, 
pan de mayz" (Valdivia, Vocabulario); "Uminta - un gisado [sic] de maíz" (Febrés, Calepino: 669). La palabra se emplea en español.

wachu /waču/. (De wakchu) adj. Ilegítimo, (animal) manso. A) "WAHCHU, adj. Huérfano, sin padres, hijo que perdió al progenitor o a la madre. Pobre, desvalido. Dícese del animal cuya madre desaparece. Solo, abandonado, expósito [...]" (Lira: 1074); "wakchu. adj. Animal huérfano de madre. EJEM: wakchu llamacha, llamita huérfana" (DQEQ: 708). B) "Huachu - el guacho, ò ilegítimo: tómase por cosa mansa en los animales" (Febrés, Calepino: 503); "HUACHU. Ilejítimo: cosa mansa en los animales" (Febrés \& Hernández I: 27). La palabra dejó de usarse; se emplea habitualmente en español, referida a personas (un hijo guacho) y animales (un cordero guacho).

wampu /wampu/. (De wampu) s. Embarcación, navío, canoa; atáud. A) "Huambo - canoa, naue de vn madero. Huambo - nauio, o balsa de madera" (Santo Tomás II: 284); "Huampu. Nauio balsa, o barco" (González Holguín I: 175); "Canoa. Huampu" (González Holguín II: 445); "wampu, s. el buque, bote, la balsa" (Middendorf: 428); "WAMPU. Canoa, barco, navío, balsa, piragua [...]" (Perroud \& Chouvenc II: 187); “wamp'u. s. Balsa de palos hecha de madera ligera [...]" (DQEQ: 715). B) "Huampu, varco o nauio" (Valdivia, Vocabulario); "Wampo scapha, A Boat, ein Boht [sic], una canoa (bote)" (Schuller: 9, 36, 53); "Huampu - cualquiera embarcación. Huamputun -hacerla o andar en ella, navegar o manejarla, trabajando, o pasando [¿paseando?] con ella" (Febrés, Calepino: 504-505); "Huampu, linter, navis, navigium. Huamputun, navigare; navem, lintrem construere" (Havestadt II: 670); "HUAMPU. Cualquiera embarcación: huampun, hacerla" (Febrés \& Hernández I: 27); "wampo, wampu, s. la canoa [...]// -tun, n., navegar, pasearse en canoa" (Augusta I: 245); "wampu, wanpu la canoa, el ataúd, la urna. wampotun navegar, pasearse en canoa. wampon hacer canoa, construir canoa, hacer urna" (Alonqueo 1989: 203). El ataúd o urna mortuoria era hecho/a de un tronco de árbol ahuecado; actualmente ya no se emplea.

warangka /wa.sanka/. (De waranqa) adj. Mil. A) "Huaranga - mil en número" (Santo Tomás II: 286); "Huaranca. Mill” (González Holguín I: 182); "huaranka. Mil" (Middendorf: 437); "WARANJA. 1. mil" (Perroud \& Chouvenc II: 190); "waranqa. adj. num. card. Mil (1000) millar, mil unidades" (DQEQ: 723). B) "Huaranca. 1000" (Valdivia, Arte, fol. 49); "Huaranca - mil [...]" (Febrés, Calepino: 505); "Huaranca, mille" (Havestadt II: 670); "HUARANCA. Mil” (Febrés \& Hernández I: 27); "waranka [warangka] /, núm. Mil" (Augusta I: 246); "M 1000 (kiñe) waranka mil" (Alonqueo 1989: 53). 
warku /wa.sku/. (De warku) s. Peso (moneda), balanza. A) "Huarco, o balanza de peso" (Santo Tomás II: 286); "Huarcu. El peso, Huarco collque. Peso, pesa de plata" (González Holguín I: 182); "Peso de plata. Ccollque ccollque huarco. De oro Ccori huarco. Peso de valanças. Huarcu" (González Holguín II: 629); "huarcu, s. la pesa, la cosa pesada, un cierto peso en plata; el peso español, moneda del Perú" (Middendorf: 438); "WÁRKU [...] f. Moneda equivalente a un peso o sea 80 centavos actuales [1945]" (Lira: 1110); "WARKU. Peso, romana, balanza [...]" (Perroud \& Chouvenc II: 190); "warku. s. // Moneda equivalente a un peso o sea 80 centavos actuales (J. L. P.)" (DQEQ: 725). B) "Quiñe úlmen eúenbi ta quiñe hueque quiñe Huinca, mari huarcu meu vn cacique vendió a vn español una oueja de la tierra por diez pesos" (Valdivia, Arte, fol. 40). La palabra dejó de usarse.

wayna /waina/. (De wayna) s. Hombre joven. A) "Guayna - moço o mancebo" (Santo Tomás II: 283); "Huayna. Moço, mancebo [...]” (González Holguín I: 193); "huaina, s., adj., joven, el joven, mozo; el amante, galán" (Middendorf: 405); "WAINA. 1. Joven, mozo, mancebo" (Perroud \& Chouvenc II: 185); "wayna. s. Joven, mozo, adolescente, hombre de 18 a 30 años" (DQEQ: 735). B) "Huayna - mozito que le apunta el bozo" (Valdivia, Vocabulario). La palabra dejó de usarse. No se registra en los otros léxicos del mapudungu(n). Actualmente se dice weche wentru /weče went ${ }^{r} \mathbf{u} /$ 'hombre joven'. Wayna se incorporó al español rural.

wincha /winča/. (De wincha) s. Cinta, fajita (para amarrar el cabello). A) "Uincha - cofia de mujer" (Santo Tomás II: 369); "Vincha. Cinta de indias que traen en la cabeça, o apretador de los cabellos" (González Holguín I: 353); "huincha, s. cinta de lana con que las mujeres se contienen el pelo" (Middendorf: 461); "WINCHA, f. Cinta usada por las nubendas a manera de faja que rodeaba la frente, con un rozón o lazada en la parte posterior. Ajustador de los cabellos usado por las mujeres" (Lira: 1156); "WINCHA. 1. Cinta para sujetar los cabellos usada por las mujeres" (Perroud \& Chouvenc II: 194); "wincha. s. Cintillo que rodea el sombrero" (DQEQ: 749). B) "Huyncha, traçadera de cabeça" (Valdivia, Vocabulario); "Huincha - faxita angosta de lana, que les sirve de trenzadera para amarrar los Cabellos" (Febrés, Calepino: 514 - 515); "Huincha, taeniola, vitta, crinales fasciolae" (Havestadt II: 682); "HUINCHA [...] fajita angosta de lana que les sirve de trenzadera para amarrar el cabello" (Febrés \& Hernández I: 31). La palabra dejó de usarse. Se la emplea habitualmente en español, con el significado de 'cinta (de género, cartulina, plástico, metal etc.)' que se usa para atar, embalar, adornar, etc.), 'cinta marcada en centímetros y pulgadas que sirve para medir (huincha de medir)'. 
yana /jana/. (De yana) s. Criado, indígena de servicio. A) "Yananc - page o criado generalmente" (Santo Tomás II: 298); "Yana, o pachaca. Criado moço de seruicio" (González Holguín I: 363); "Yanacuna. Los criados, o vn criado [...]" (González Holguín I: 364); "yana, s. el compañero, sirviente [...] yanacuna la servidumbre" (Middendorf: 107); "YANA. 3. Criado, mozo de servicio" (Perroud \& Chouvenc II: 197); "yana. s. [...]// Mozo o moza de servicio o criado [...]" (DQEQ: 759). B) "Yana, criado" (Valdivia, Vocabulario); "Yana - el criado, ò paje, ò servicio de Españoles: Yana cona - los Indios ladinos criados entre Españoles" (Febrés, Calepino: 524); "Yana, yanacona, famulus, servus" (Havestadt II: 685); "YANA. Indios ladinos, criados entre españoles" (Febrés \& Hernández I: 35), "yana (kon·a)*, los indios ladinos criados entre los españoles" (Augusta I: 282); "Yan [¿yana?], yanakona, el mozo, el mozo criado por los españoles" (Alonqueo 1989: 240). Se emplea en español para referirse a una persona que adopta una actitud servil.

\section{CONCLUSIÓN}

El mapuche (mapudungu(n) -la lengua originaria de mayor difusión en Chile, en el pasado y en presente- adoptó, seguramente desde la época precolombina, léxico del idioma quechua, referido a diferentes campos semánticos. Algunos han dejado de usarse; otros se mantienen vigentes en la lengua hablada en la actualidad. Ya en la época colonial dieron origen a léxico derivado, sobre todo verbos. Parte del léxico quechua se incorporó también al español, el cual se ha usado habitualmente.

\section{REFERENCIAS BIBLIOGRÁFICAS}

Academia Chilena de la Lengua. 2010. Diccionario de uso del español de Chile (DUECh). Santiago, Chile: MN Editorial Ltda.

Academia Mayor de la Lengua Quechua. 1995. Diccionario Quechua-Español-Quechua. Qheswa-Español-Qheswa Simi Taqe. Qosqo: Municipalidad del Qosqo.

Alonqueo, MarTín. 1985. Mapuche ayer - hoy. Padre Las Casas, Chile: Imprenta y Editorial "San Francisco".

1989. El habla de mi tierra. Mapudungun. P. Las Casas, Temuco: Ediciones Kolping. 
Augusta, Félix José DE. 1916. Diccionario Araucano-Español y Español-Araucano. Tomos I y II. Santiago de Chile: Imprenta Universitaria.

Catrileo, María. 1996. Diccionario Lingüístico-Etnográfico de la Lengua Mapuche. Mapudungun-Español-English. Segunda edición. Santiago - Chile: Editorial Andrés Bello.

Encina, Francisco A. 1983. Historia de Chile. Texto original completo. Tomo I. Santiago: Editorial Ercilla.

Febrés, Andrés. 1765. Arte de la Lengua General del Reyno de Chile, con un Diálogo ChilenoHispano muy curioso: a que se añade la Doctrina Christiana, esto es, Rezo, Catecismo, Coplas, Confesionario, y Pláticas; lo más en Lengua Chilena y Castellana: y por fin un Vocabulario Hispano- Chileno, y un Calepino Chileno-Hispano más copioso. Compuesto por el P. Andrés Febres Misionero de la Comp. de Jesus. Año de 1764 [...]. Con licencia: en Lima, en la calle de la Encarnación. Año de 1765.

y Antonio Hernández. 1846. Diccionario Chileno-Hispano, compuesto por el R.P. Andrés Febrés De la C. de J. Enriquecido de voces i mejorado por el R.P. Misionero Fr. Antonio Hermández i Calzada de la orden de la Regular Observancia de N. P. S. Francisco [---]. Santiago: Imprenta de los Tribunales.

González Holguín, Diego. [1608] 1952. Vocabulario de la lengua general de todo el Perú llamada Lengua Qquichua o del Inca. Segunda edición. Prólogo de Raúl Porras Barrenechea. Lima: Universidad Nacional Mayor de San Marcos.

Havestadt, Bernardo. [1777] 1883. Chilidúǵu sive Tractatus Linguae Chilensis opera Bernardi Havestadt. Editionem novam immutatam curavit Dr. Julius Platzmann. Vols. I y II. Lipsiae. In Aedibus B. G. Teubneri.

Lenz, Rodolfo. 1905-1910. Diccionario etimolójico de las voces chilenas derivadas de lenguas indijenas americanas: Santiago de Chile: Imprenta Cervantes.

LiRA, Jorge A. 1945. Diccionario Kkechuwa - Español [...]. Tucumán - República Argentina: Universidad Nacional de Tucumán.

Middendorf, ERnst. 1890. Wörterbuch des Runa Simi oder der Keshua Sprache. Leipzig: F. A. Brockhaus.

Perroud, Pedro Clemente y Juan María Chouvenc. 1970. Diccionario Castellano Kechwa Kechwa Castellano. Dialecto de Ayacucho. Perú: Seminario San Alfonso: Padres Redentoristas.

SÁnchez, Gilberto. 2013. "Los quechuismos en el español de Chile”. En Lexicología y lexicografia en Hispanoamérica. Homenaje a Martha Hildebrandt. Marco Martos Carrera / Gladys Flores Heredia (editores). 1 ${ }^{\text {a }}$. ed. Lima: Fondo Editorial de la Academia Peruana de la Lengua, pp. 83-106.

SAnto Tomás, Domingo DE. [1560] 1951. Lexicón o vocabulario de la lengua general del Perú. Edición facsimilar, publicada con un prólogo, por Raúl Porras Barrenechea. Lima: Universidad Mayor de San Marcos.

Schuller, R. R. 1907. El Vocabulario Araucano (de 1642-1643). Bibliografía de la Lengua Mapuche o Araucana. Santiago de Chile: Imprenta Cervantes.

VAldivia, LuIs DE. 1606. Arte y Gramática General de la Lengva Que corre en todo el Reyno deChile, con vn Vocabulario, y Confessonario. Compuestos por el Padre Luys de Valdiuia de la Compañia de Iesus en la Prouincia del Piru [...] En Lima por Francisco del Canto. Año. 1606.

VIVAR, GERÓNIMO DE. [1558] 1979. Crónica y relación copiosa y verdadera de los Reynos de Chile. Edición de Leopoldo Sáez-Godoy. Bibliotheca Ibero Americana. Veröffentlichungen des Ibero-Amerikanischen Instituts Preussischer Kulturbesitz. Band 27. Berlin: Colloquium Verlag. 